\title{
CORTICAL VOLUME IS INVERSELY CORRELATED WITH INTERLEUKIN-6 SERUM LEVELS IN PATIENTS WITH SCHIZOPHRENIA
}

Clin Biomed Res. 2021;41(3):1-223

1 Departamento de Radiologia e Ressonância Magnética, Hospital de Clinicas de Porto Alegre (HCPA). Porto Alegre, RS, Brasil.

2 Departamento de Psiquiatria, Universidade Federal do Paraná (UFPR). Curitiba, PR, Brasil.

3 Laboratório de Psiquiatria Molecular, Hospital de Clínicas de Porto Alegre (HCPA). Porto Alegre, RS, Brasil.

Corresponding author: Sandra Lermen Polita spolita@hcpa.edu.br Hospital de Clínicas de Porto Alegre (HCPA)

Rua Ramiro Barcelos, 2350

90035-007, Porto Alegre, RS, Brasil.

\section{Sandra Lermen Polita ${ }^{1}$, Raffael Massuda², Bruna Panizzutti ${ }^{3}$, Clarissa S. Gama ${ }^{3}$, Juliana Avila Duarte ${ }^{1}$}

\section{ABSTRACT}

Introduction: It is well established that cortical volume are decreased in patients with schizophrenia. One possible explanation is that the increased pro-inflammatory status in schizophrenia is related to volumetric decrease of gray matter. The aim of this study was to correlate interleukin 6 (IL-6) with cortical volume in patients with schizophrenia and controls.

Methods: We selected 36 patients with schizophrenia and 35 controls. Interleukin 6 (IL-6) was correlated with cortical volume in patients with schizophrenia and controls.

Results: IL-6 was negatively correlated with cortical volume $(p=0.027$; rho $=-0.370)$ in patients, but not in controls $(p=0.235)$.

Discussion: Our results are in line with previous studies suggesting that chronic inflammatory activation in patients with schizophrenia could be one plausible mechanism that could contribute for the cortical volumetric decrease often seen in this population. However, this cross-sectional study with a small number of patients does not allow us to establish causal relations.

Keywords: Schizophrenia; Inflammation; MRI

\section{INTRODUCTION}

Schizophrenia may be associated with progressive enlargement of brain ventricles ${ }^{1}$. Although not conclusive, structural deficits noted in studies include increased lateral ventricular volumes, decreased frontal lobes and thalamic volumes, grey matter loss within the anterior cingulate, superior temporal gyrus and hippocampus, and decreased overall cerebral size ${ }^{2}$. The progressive volume changes in temporal and frontal lobes observed in patients with schizophrenia are striking in light of cross-sectional magnetic resonance imaging (MRI) studies showing particularly prominent volume deficits in these regions. Decline in temporal lobe tissue or gray matter was observed in patients with childhood-onset and first-episode schizophrenia ${ }^{3}$. The hippocampal volume of anyone is the result of several features; for instance, the presence and severity of comorbidities (like diabetes mellitus), as well as therapeutic measures aiming to get better fitness, can enlarge the size of the hippocampus. Besides that, the size of the hippocampus, as much as the whole brain, is associated with individual's height - the intracranial contents are scaled to the size of the cranial cavity. Many medical conditions can influence the size of the hippocampus with ageing, including vascular risk factors and cardiovascular disease, as well as other common diseases like traumatic brain injury and clinical depression. The pronounced cognitive decline observed in patients after cardiac arrest, obesity, diabetes mellitus, atrial fibrillation, hypertension, or obstructive sleep apnea can be related to hippocampal atrophy. Usually, multiple vascular risk factors are present in the 
same patient and can have synergistic outcomes in the normal ageing of the brain. Nevertheless, a single risk factor can also influence cognitive function through a reduction in the size of the whole brain, and the hippocampus in particular. To exemplify, children with heart failure have a great reduction in hippocampal volume without obesity, hypertension, and other vascular comorbidities ${ }^{4}$.

Cytokines regulate inflammation and coordinate both innate and adaptive arms of the immune system, being important mediators of the cross-talk between the central nervous system and the immune system, which might have implications for clinical psychiatry ${ }^{5}$.

Examples of cytokines include interleukins (IL) and tumor necrosis factors (TNF). IL-1, IL-6 and TNF-alpha are considered pro-inflammatory, in the sense that they augment the immune response to infection and inflammation by promoting leukocyte recruitment to inflammatory sites and/or by activating inflammatory cells ${ }^{6}$. IL-10 is an anti-inflammatory cytokine that contributes to dampen the immune and inflammatory response ${ }^{6}$. Thiobarbituric acid-reactive substances is one of the well-known secondary products of lipid peroxidation and was used as an indicator of oxidative damage for several diseases ${ }^{7}$.

A chronic inflammatory state with increased levels of pro- inflammatory cytokines and altered levels of Th-1-like and Th-2-like cytokines may characterize schizophrenia ${ }^{8}$. Hypothesized signs of inflammation and monocyte activation include increased levels of pro-inflammatory cytokines, e.g. TNF- $\alpha$, IL-1 and IL-6, acute phase proteins, and complement factors ${ }^{9}$.

Oxidative damage is a mechanism of cellular injury in a number of conditions, including cancer, inflammatory states, and neurodegeneration ${ }^{10}$. Increased neuronal oxidative stress levels produce deleterious effects on signal transduction, structural plasticity, and cellular resilience, mostly by inducing lipid peroxidation in membranes and direct damage in protein and genes ${ }^{11}$. Neurons and glia are particularly vulnerable to inflammatory processes and redox status, and are dependent on the maintenance of neurotrophic activity ${ }^{12}$. Furthermore, oxidative stress has been identified as a possible element in the neuropathological processes of schizophrenia ${ }^{13}$.

IL-6 has been associated with brain anatomical abnormalities in animal studies. Modeling the association between prenatal infection/inflammation and schizophrenia-related disorders in rodent models of prenatal immune activation has yielded a plethora of experimental data which strongly support the utility of these models in preclinical schizophrenia research. The poly $(\mathrm{I}: \mathrm{C})$ model in mice and rats is one of the currently available prenatal immune activation models which holds strong promise in this research area. The heuristic value of the poly $(\mathrm{I}: \mathrm{C})$ model for preclinical schizophrenia research is supported because it successfully accounts for several aspects of schizophrenia epidemiology, pathophysiology, symptomatology and treatment ${ }^{14}$. The aim of this study was to correlate interleukin 6 (IL-6) with cortical volume in patients with schizophrenia and controls.

\section{METHODS}

Seventy-one subjects were enrolled to this study protocol, 36 patients with schizophrenia (28 males; mean age $37.17 \pm 12.05$; years of disease $15.56 \pm 11.75$ ) and 25 controls ( 21 males, mean age $36.97 \pm 13.04$ ). Inclusion criteria for patients and controls have been described elsewhere ${ }^{15}$. All patients received pharmacological treatment by their psychiatrist according to clinical protocols. Written informed consent was obtained from all subjects in accordance with the Declaration of Helsinki and the local ethics committee approved the study protocol.

The Structured Clinical Interview for DSM-IV (SCID) Axis I and Axis II was administered to confirm diagnosis. Sociodemographic, clinical and pharmacological data were collected via a structured interview with the patient and examination of clinical records. Trained raters assessed the psychopathological status by the 18-item Brief Psychiatric Rating Scale (BPRS) ${ }^{16}$. Each subject had $5 \mathrm{~mL}$ blood samples collected by venipuncture without anticoagulants, and serum was obtained by centrifugation at $300 \mathrm{~g}$ for $5 \mathrm{~min}$ and kept frozen at $70^{\circ} \mathrm{C}$ for up to 6 months, until the assay. Serum IL- 6 was measured according to the procedures supplied by the manufacturer using highly sensitive sandwich-ELISA kits for IL-6 (Quantikine, R\&D Systems, Minneapolis, USA). All samples were assayed in duplicates.

T1-weighted magnetic resonance images were acquired with a Philips Achieva 1.5 Tesla scanner (Amsterdam, the Netherlands). The cortical volumetric segmentations were performed with the Freesurfer image analysis suite v.5.1.0, (http://surfer.nmr.mgh. harvard.edu/), an automated software that allows the MRI measurement of cortical and intracranial volumes ${ }^{17}$. All images were processed and checked by the same researcher. Intracranial volume was regressed out from total cortex volume.

Demographic and clinical characteristics were analyzed using chi-square, Mann-Whitney or $t$-test. The correlation between cortex volume and IL-6 serum levels was obtained by Spearman-rank correlation coefficient.

\section{RESULTS}

Demographic data and comparisons between groups are shown in Table 1. IL-6 was negatively correlated with cortical volume $(p=0.027$; rho $=-0.370)$ in patients but not in controls $(p=0.235)$. 
Polita et al.

Table 1: Characteristics of healthy controls and patients with schizophrenia.

\begin{tabular}{|c|c|c|c|}
\hline & Healthy controls $(n=35)$ & $\begin{array}{c}\text { Patients with } \\
\text { schizophrenia }(n=36)\end{array}$ & p-value \\
\hline Gender (male/female) & $21 / 14$ & $28 / 8$ & $0.11^{\mathrm{b}}$ \\
\hline $\mathrm{Age}^{\mathrm{a}}$ & $36.97(13.04)$ & $37.17(12.05)$ & $0.94^{c}$ \\
\hline Years of education ${ }^{a}$ & $10.86(2.54)$ & $10.42(3.16)$ & $0.52^{c}$ \\
\hline & 475.62 & 439.60 & \\
\hline Cortical volume $\left(\mathrm{cm}^{3}\right)^{d}$ & $(467.66-483.58)$ & $(431.75-447.46)$ & $<0.001$ \\
\hline IL-6 & $7.51(1.43)$ & $6.99(1.99)$ & $0.61^{f}$ \\
\hline Intracranial volume $\left(\mathrm{cm}^{3}\right)$ & $1587.86(141.16)$ & $1583.58(166.27)$ & $0.91^{\mathrm{c}}$ \\
\hline $\begin{array}{l}\text { Correlation of cortex volume and IL-6 } \\
(\mathrm{pg} / \mathrm{mL})^{g}\end{array}$ & -0.206 & $-0.370^{*}$ & \\
\hline
\end{tabular}

${ }^{a}$ Mean (standard deviation); ${ }^{\mathrm{b}}$ Chi-square; ${ }^{\mathrm{c}} T$-test; ${ }^{\mathrm{d}}$ ANCOVA with estimated mean ( $95 \%$ confidence interval). Covariates: age, years of education, and intracranial volume; e Median (interquartile range); ${ }^{\dagger}$ Mann-Whitney test; ${ }^{9}$ Intracranial volume was regressed out from cortical volume. Spearman correlation (rho).

$* p<0.05$

\section{DISCUSSION}

In our study, we found a correlation between IL-6 and cortical volume in patients with schizophrenia. Increased pro-inflammatory tonus has been one theory for the gray matter loss in schizophrenia. Many studies highlight the importance of IL-6 as a protagonist in this inflammatory scenario, at least in acute phases ${ }^{5}$. There are some limitations in the present study. It is a cross-sectional study; longitudinal studies are needed to correlate inflammation to cortical volume. An important limitation of our study is that we did not correlate brain volumes and IL- 6 levels with cigarette smokers, although cross-sectional brain imaging studies suggest that healthy subjects who smoke cigarettes show smaller gray matter volumes and/or densities in the prefrontal, anterior cingulate, occipital, and temporal cortices (including parahippocampal gyrus), thalamus, substantia nigra and cerebellum as compared to non-smokers ${ }^{18}$. Our results are in line with previous studies suggesting that chronic inflammatory activation in patients with schizophrenia could be one plausible mechanism that could contribute for the cortical volumetric decrease often seen in this population. However, this crosssectional study with a small number of patients does not allow us to establish causal relations.

\section{Acknowledgment}

This study was supported by CAPES, FAPERGS and CNPq (Brazil).

\section{REFERENCES}

1. Van Haren NE, Cahn W, Hulshoff Pol HE, Kahn RS. The course of brain abnormalities in schizophrenia: can we slow the progression? J Psychopharmacol. 2012;26(5 suppl):8-14.

2. Andreasen NC, Nopoulos $P$, Magnotta V, Pierson R, Ziebell S, Ho BC. Progressive brain change in schizophrenia: a prospective longitudinal study of first-episode schizophrenia. Biol Psychiatry. 2011;70(7):672-9.

3. Mathalon DH, Sullivan EV, Lim KO, Pfefferbaum A. Progressive brain volume changes and the clinical course of schizophrenia in men: a longitudinal magnetic resonance imaging study. Arch Gen Psychiatry. 2001;58(2):148-57.
4. Fotuhi M, Do D, Jack C. Modifiable factors that alter the size of the hippocampus with ageing. Nat Rev Neurol. 2012;13;8(4):189-202.

5. Pedrini M, Massuda R, Fries GR, Pasquali MAB, Schnorr CE, Moreira JCF, et al. Similarities in serum oxidative stress markers and inflammatory cytokines in patients with overt schizophrenia at early and late stages of chronicity. J Psychiatr Res. 2012;46(6):819-24.

6. Potvin S, Stip E, Sepehry AA, Gendron A, Bah R, Kouassi E. Inflammatory cytokine alterations in schizophrenia: a systematic quantitative review. Biol Psychiatry. 2008;63(8):801-8.
7. Huang TL, Liou CW, Lin TK. Serum thiobarbituric acid-reactive substances and free thiol levels in schizophrenia patients: effects of antipsychotic drugs. Psychiatry Res. 2010;177(1-2):18-21.

8. Miller BJ, Buckley P, Seabolt W, Mellor A, Kirkpatrick B. Metaanalysis of cytokine alterations in schizophrenia: clinical status and antipsychotic effects. Biol Psychiatry. 2011;70(7):663-71.

9. Mayilyan KR, Arnold JN, Presanis JS, Soghoyan AF, Sim RB. Increased complement classical and mannanbinding lectin pathway activities in schizophrenia. Neurosci Lett. 2006;404(3):336-41. 
10. Kapczinski F, Dal-Pizzol F, Teixeira AL, Magalhaes PV, Kauer-Sant'Anna M, Klamt F, et al. Peripheral biomarkers and illness activity in bipolar disorder. J Psychiatr Res. 2001;45(2):156-61.

11. Gama CS, Andreazza AC, Kunz M, Berk M, Belmonte-de-Abreu PS, Kapczinski F. Serum levels of brain-derived neurotrophic factor in patients with schizophrenia and bipolar disorder. Neurosci Lett. 2007;420(1):45-8.

12. Kapczinski F, Dal-Pizzol F, Teixeira AL, Magalhaes PV, Kauer-Sant'Anna M, Klamt F, et al. A systemic toxicity index developed to assess peripheral changes in mood episodes. Mol Psychiatry. 2010;15(8):784-6.
13. Riegel RE, Valvassori SS, Moretti M, Ferreira CL, Steckert AV, Souza B, et al. Intracerebroventricular ouabain administration induces oxidative stress in the rat brain. Int J Dev Neurosci. 2010;28(3):233-7.

14. Meyer U, Feldon J. To poly(I:C) or not to poly $(\mathrm{I}: \mathrm{C})$ : advancing preclinical schizophrenia research through the use of prenatal immune activation models. Neuropharmacology. 2012;62(3):1308-21.

15. Czepielewski LS, Massuda R, Goi P, Sulzbach-Vianna M, Reckziegel R, Costanzi M, et al. Verbal episodic memory along the course of schizophrenia and bipolar disorder: a new perspective. Eur Neuropsychopharmacol. 2015;25(2):169-75.

16. Romano F, Elkis H. Tradução e adaptação de um instrumento de avaliação psicopatológica das psicoses: a escala breve de avaliação psiquiátrica-versão ancorada (BPRS-A). J Bras Psiquiatr. 1996;45(1):43-9.

17. Fischl B. FreeSurfer. Neuroimage. 2012;62(2):774-81.

18. Van Haren NE, Koolschijn PC, Cahn W, Schnack HG, Hulshoff Pol HE, Kahn RS. Cigarette smoking and progressive brain volume loss in schizophrenia. Eur Neuropsychopharmacol. 2010;20(7):454-8.

Received: Aug 24, 2020 Accepted: May 1, 2021 